\title{
Analysis of the long-term beneficial effects of menopausal hormone therapy on sleep quality and menopausal symptoms
}

\author{
CAIXIA LI, LI WANG, XIAOHUA SUN and XIAOMEI YANG
}

\author{
Department of Obstetrics and Gynecology, \\ Maternity and Child Health Care of Zaozhuang, Zaozhuang, Shandong 277100, P.R. China
}

Received August 19, 2019; Accepted September 23, 2019

DOI: $10.3892 /$ etm.2019.8058

\begin{abstract}
A large number of menopausal women report sleep disturbances along with psychological, somatic and urogenital menopausal symptoms. The aim of this study was to evaluate the efficacy of menopausal hormonal therapy (MHT) in improving subjective sleep quality and the severity of menopausal symptoms. An institutional ethics committee approved this retrospective chart review of 342 women treated with MHT for menopausal symptoms. Standard 28-day MHT consisted of the oral administration of $2 \mathrm{mg}$ estradiol daily for 14 days, followed by $2 \mathrm{mg}$ estradiol and $10 \mathrm{mg}$ dydrogesterone daily for the remaining 14 days. A subgroup of 14 participants with a family history of cancer and mammography scores of 3 and above, received only tibolone $2.5 \mathrm{mg}$ daily. Perceived sleep quality was measured with the Pittsburgh Sleep Quality Index (PSQI), while the assessment of menopausal symptoms was performed using the Kupperman Menopause Index (KMI) and menopause rating scale (MRS). Of the 342 patients, 79 were followed-up for 3 years. Compared to the baseline scores, the mean decrease in PSQI scores was $1.53 \pm 0.29$ points $(\mathrm{P}<0.0001)$ at 1 month, $2.21 \pm 0.187$ points $(\mathrm{P}<0.0001)$ at 2 months and $2.26 \pm 0.6$ points $(\mathrm{P}<0.0001)$ after 3 years of MHT. The KMI scores also decreased by a mean of $6.37 \pm 1.59$ points $(\mathrm{P}<0.0001)$ at 1 month and by $8.73 \pm 1.92$ points after 3 years $(\mathrm{P}<0.0001)$. The MRS scores decreased by a mean of $3.56 \pm 1.05$ points $(\mathrm{P}<0.0001)$ at 1 month and by $4.28 \pm 2.01$ points $(\mathrm{P}<0.0001)$ after 3 years, as compared to the baseline scores. Patients receiving tibolone MHT did not report any improvement in sleep quality $(\mathrm{P}=0.956)$. On the whole, the findings of this study indicate that conventional MHT has a rapid and prolonged beneficial effect on self-reported sleep quality and menopausal symptoms in women. However, further clinical
\end{abstract}

Correspondence to: Dr Xiaomei Yang, Department of Obstetrics and Gynecology, Maternity and Child Health Care of Zaozhuang, 25 Wenhua East Road, Zaozhuang, Shandong 277100, P.R. China E-mail: xmyang2019@163.com

Key words: hormone treatment, menopausal symptoms, femoston, tibolone, Pittsburgh Sleep Quality Index (PSQI) studies are warranted to compare the effects of different MHT regimens.

\section{Introduction}

Menopause is defined as the end of a woman's reproductive period, associated with the decreased production of estradiol and progesterone by the ovaries (1). With increased life expectancies worldwide, it is estimated that by 2025, the number of post-menopausal women will reach 1.1 billion (2). The gradual or sudden cessation of ovarian follicular function can manifest in a number of psychological, somatic and urogenital menopause symptoms that have a significant impact on the quality of life of women (3). These severity of climacteric complaints is routinely determined by using the Kupperman Menopause Index (KMI) and the menopause rating scale (MRS) $(4,5)$ that evaluate menopause-related somato-vegetative, psychological and urogenital symptoms (6).

Insomnia is considered one of the most common symptoms of menopause, occurring in over half of climacteric women (7-11). Chronic poor sleep has the most profound negative effect on the quality of life of menopausal women (12-14), and can also lead to the development and exacerbation of cardiovascular and metabolic diseases (15). Therefore, addressing emerging sleep symptoms during menopause may significantly improve the overall health and wellbeing of women.

Systemic estrogen/progestin menopausal hormone therapy (MHT) is one of the most common treatments used to counteract menopausal symptoms, such as hot flashes, night sweats and urogenital atrophy (16). However, in women with a previous history of breast cancer, conventional estrogen/progestin MHT may increase the risk of recurrence. This risk may be reduced by the use of progestogens, and among these, is the synthetic steroid tibolone, whose metabolites have estrogenic, progestogenic and androgenic properties (17). The effect of MHT on sleep quality remains poorly understood. Several randomized clinical trials have demonstrated that estrogen/progestin MHT results in slightly improved sleep quality in menopausal women with vasomotor symptoms $(18,19)$. However, significant heterogeneity in the selection criteria of the trial participants and formulations of MHT make it is difficult to determine whether the same effect could be achieved in general population of menopausal women (19). 
Sleep disturbances can be either evaluated by objective methods such as polysomnography, or by using patient-filled questionnaires, such as the self-rated Pittsburgh Sleep Quality Index (PSQI) that assesses sleep quality and disturbances over a time interval $(20,21)$. A systematic review by Devine et al demonstrated that questionnaires filled by the patient are an accurate tool for predicting the quality of sleep, and evaluating the efficacy of different treatments (22).

In this study we use PSQI to assess the effects of estrogen/progestin and progestogen MHT on sleep quality, and further examined the effects of MHT on the overall quality of life of women experiencing menopausal symptoms.

\section{Subjects and methods}

Study design and participants. The Ethics Committee of the Maternity and Child Health Care of Zaozhuang approved this retrospective medical record review (approval no. 2016006). From the history reports of the patients who were enrolled in this study, all patients or patient carers signed informed consents. A total of 342 women newly attending the outpatient clinic who were naïve to MHT treatment were enrolled in this study. The menopausal status of the participants was assessed by their self-reported menstrual history. Menopause was determined as the cessation of menstruation with 12 months of amenorrhea. All participants were required to fill in a demographic form, as well as detailed medical history, including the characteristics of menopause, urogenital and metabolic disorders, and any previous history of oncological diseases. The study was approved by the ethics review board of the hospital and each participant provided their written informed consent prior to participation.

MHT. All participants enrolled in this study received MHT. Women with no family history of breast cancer and mammography scores $<3$ received femoston (estradiol/dydrogesterone 2/10 mg, Abbott laboratories) according to the established standard protocol. Briefly, 1 red tablet, containing $2 \mathrm{mg}$ estradiol was administered daily for the first 14 days, and 1 yellow tablet, containing $2 \mathrm{mg}$ estradiol and $10 \mathrm{mg}$ dydrogesterone was administered daily for the following 14 days of each 28-day cycle orally. A subgroup of 14 participants with a family history of cancer and mammography scores $\geq 3$, received tibolone (Livial $^{\circledR}$; Organon), $2.5 \mathrm{mg}$ daily.

Determination of sleep quality and severity of menopausal symptoms. Perceived sleep quality was measured and scored with the PSQI, as previously described (23). A total score $>5$ indicates insufficient sleep quality; a PSQI $\leq 5$ indicates good sleepers, while a PSQI $>5$ corresponds to poor sleepers. The assessment of menopausal symptoms was performed using the KMI and the MRS that measure the severity of age-/menopause-related complaints by rating a profile of symptoms and their impact on health-related quality of life.

Statistical analysis. All variables are expressed as the means \pm standard deviation. Follow-up data at each time interval was compared using a one-way analysis of variance test (ANOVA) followed by Tukey's honestly significant differ- ence (HSD) test. Values of $\mathrm{P}<0.05$ were considered to indicate statistically significant differences.

\section{Results}

This study comprised of 342 women with an average age (SD) of 50.3 (5.6) years. The baseline demographic and medical characteristics for all the subjects are summarized in Table I. The mean weight of the participants was 56.5 (7.3) $\mathrm{kg}$ and the average age at the onset of menses was 14.3 (1.6) years. The majority of women enrolled in this study (95.9\%) had no family history of breast disease. In addition, the majority of women did not suffer from or were treated for cervical, endometrial or ovarian cancer prior to exhibiting menopausal symptoms $(100,99.7$ and $100 \%$, respectively). The majority of women did not have a confirmed diagnosis of endometriosis and uterine fibroids (99.4 and $95.9 \%$ respectively), and had not undergone any type of urogenital surgical interventions (98.8\%). The majority of participants also had no previous history of hypertension $(93.9 \%)$, diabetes $(98.8 \%)$, lipid metabolism disorders $(94.2 \%)$, gallbladder disease $(94.4 \%)$ or thyroid dysfunction (90.6\%).

At the start of the study (baseline), the mean PSQI score of the participants was 9.16 (4.84) points, indicative of a poor sleep quality (Table II). At 1 month after the commencement of beginning MHT, the participants reported significantly improved sleep quality as indicated by the reduction in the PSQI global sleep quality score (decrease of 1.53 points; $\mathrm{P}<0.0001$ ), as compared to the baseline score. The PSQI score further decreased at 2 months after the commencement of MHT (2.21 points, $\mathrm{P}<0.0001)$, and continued to remain significantly below baseline scores throughout the 3 years of continuous treatment $(\mathrm{P}<0.0001)$. After 3 years of MHT, the participants continued to maintain a mean decrease (SD) of $2.19(0.21)$ points (1.3-fold) as compared to the baseline score (Fig. 1).

The mean (SD) KMI score of the participants at the baseline was 18.63 (9.86) (Table III). We observed a 6.37 (1.59) point decrease in the score during the 1st month of MHT $(\mathrm{P}<0.0001)$. The KMI scores continued to gradually decline throughout the course of hormone therapy, reaching an average of 9.9 (7.33), with a decrease of 8.73 (1.92) points compared to the baseline score $(\mathrm{P}<0.0001$; Fig. 2$)$.

There was a significant [3.56 $(1.05)$ points $(\mathrm{P}<0.0001)]$ decrease in the mean MRS scores of the participants during the 1st month of MHT as compared to baseline (Fig. 3). Subsequent hormone treatment did not result in further significant changes in the MRS scores (Fig. 3), and the average score at 3 years after the commencement of MHT was 4.7 (4.6), as compared to $8.66(6.17)$ at baseline, an equivalent of 3.96 (2.01)-point decrease $(\mathrm{P}<0.0001$; Table IV and Fig. 3).

We then evaluated whether different MHT regimens (femoston or tibolone) had a different impact on the changes in the PSQI scores. In the subgroup of participants receiving femoston, the baseline mean PSQI score was 9.14 (4.84) points (Table V). During the 1st and 2nd months of MHT, the PSQI score improved significantly, exhibiting a decrease of 1.62 (1.01) $(\mathrm{P}=0.04)$, and $2.26(0.72)$ points $(\mathrm{P}=0.0002)$ respectively, comparing to baseline (Fig. 4). There was no further significant decrease in the PSQI score, maintaining a stable 
Table I. Demographic characteristics and medical history of the participants.

\begin{tabular}{|c|c|c|c|}
\hline Demographics & No. of participants & Mean & $( \pm \mathrm{SD})$ \\
\hline Age & 342 & 50.3 & 5.6 \\
\hline Weight (kg) & 325 & 56.5 & 7.3 \\
\hline Age at 1 st period & 181 & 14.3 & 1.6 \\
\hline Medical history & No. of participants & Yes $(\%)$ & No $(\%)$ \\
\hline Dysmenorrhea & 182 & $66(36.8)$ & $115(63.2)$ \\
\hline Stress before period & 182 & $33(18.1)$ & $149(81.9)$ \\
\hline Gynecological surgery & 342 & $54(15.8)$ & $288(84.2)$ \\
\hline Family history of breast disease & 342 & $14(4.1)$ & $328(95.9)$ \\
\hline History of endometrial cancer prior to onset of menopausal symptoms & 342 & $0(0.0)$ & $342(100.0)$ \\
\hline Treated for cervical cancer prior to onset of menopausal symptoms & 342 & $1(0.3)$ & $341(99.7)$ \\
\hline History of ovarian cancer prior to onset of menopausal symptoms & 342 & $0(0.0)$ & $342(100.0)$ \\
\hline Confirmed diagnosis of endometriosis & 342 & $2(0.6)$ & $340(99.4)$ \\
\hline Surgical interventions for uterine fibroids & 342 & $14(4.1)$ & $328(95.9)$ \\
\hline Gynecological benign surgery - other surgeries & 342 & $4(1.2)$ & $338(98.8)$ \\
\hline Tumor (current) & 342 & $0(0.0)$ & $342(100.0)$ \\
\hline History of hypertension & 342 & $21(6.1)$ & $321(93.9)$ \\
\hline History of diabetes & 342 & $4(1.2)$ & $338(98.8)$ \\
\hline History of lipid metabolism disorder & 342 & $20(5.8)$ & $322(94.2)$ \\
\hline History of gallbladder disease & 342 & $19(5.6)$ & $323(94.4)$ \\
\hline History of thyroid disease & 342 & $32(9.4)$ & $310(90.6)$ \\
\hline History of other diseases & 342 & $20(5.8)$ & $322(94.2)$ \\
\hline
\end{tabular}

Variables with a number $<342$ indicate missing collected data from some of the subjects. Numbers in parentheses are in percentages.

Table II. Comparison of PSQI scores of the study participants at baseline and follow-up assessments.

\begin{tabular}{|c|c|c|c|c|c|}
\hline MHT period & No. of participants & PSQI mean $( \pm \mathrm{SD})$ & $\begin{array}{l}\text { Delta-PSQI } \\
\text { mean }( \pm S D)\end{array}$ & $\begin{array}{c}\text { One-way ANOVA } \\
\text { (P-value) }\end{array}$ & $\begin{array}{c}\text { Tukey's HSD post-hoc test } \\
\text { (P-value) }\end{array}$ \\
\hline 0 days (baseline) & 342 & $9.16(4.84)$ & 0 & $\mathrm{~N} / \mathrm{A}$ & - \\
\hline 1st month & 111 & $7.63(4.58)$ & $1.53(0.29)$ & & - \\
\hline 2nd month & 108 & $6.97(4.50)$ & $2.21(0.187)$ & & $<0.0001$ \\
\hline 3rd month & 93 & $7.02(4.01)$ & $2.16(0.2)$ & & $<0.0001$ \\
\hline$>3$ months & 171 & $6.95(4.32)$ & $2.21(0.221)$ & & $<0.0001$ \\
\hline$>6$ months & 113 & $6.97(4.33)$ & $2.19(0.191)$ & $<0.0001$ & $<0.0001$ \\
\hline$>9$ months & 84 & $7.37(4.41)$ & $1.79(0.139)$ & & $<0.0001$ \\
\hline$>1$ year & 134 & $6.90(4.17)$ & $2.28(0.19)$ & & $<0.0001$ \\
\hline$>2$ years & 76 & $6.71(4.56)$ & $2.45(0.161)$ & & $<0.0001$ \\
\hline$>3$ years & 79 & $6.90(4.76)$ & $2.26(0.6)$ & & $<0.0001$ \\
\hline
\end{tabular}

All 342 subjects received hormone treatment. The PSQI scores were recorded prior to the commencement of MHT (baseline), and reassessed at varying intervals over the course of the treatment. Delta-PSQI indicates the difference between the baseline and follow-up score. Each subject's score in the follow-up assessment was matched with their own baseline score. Comparisons were made using one-way ANOVA followed by Tukey's HSD post-hoc test. $\mathrm{P}$-values at each follow-up are as compared to values at baseline, with a value of $\mathrm{P}<0.05$ considered to indicate a statistically significant difference with a value of $\mathrm{P}<0.05$ considered to indicate a statistically significant difference. Some subjects had multiple assessments during a given interval. MHT, menopausal hormonal therapy; PSQI, Pittsburgh Sleep Quality Index; N/A, not available; ANOVA, analysis of

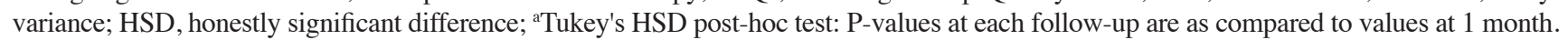

2.28 (0.67)-points difference compared to the initial score prior to the commencement of MHT $(\mathrm{P}<0.005)$.
In the small subgroup of 14 participants with a previous history of breast cancer, who were receiving tibolone MHT, no 
Table III. Changes in KMI scores of the participants receiving MHT.

\begin{tabular}{|c|c|c|c|c|}
\hline MHT period & No. of participants & Mean $( \pm$ SD) & One-way ANOVA (P-value) & Tukey's HSD post-hoc test ${ }^{\mathrm{a}}$ (P-value) \\
\hline 0 days (baseline) & 342 & $18.63(9.86)$ & \multirow{10}{*}{$<0.0001$} & - \\
\hline $1 \mathrm{st}$ month & 111 & $12.26(8.09)$ & & $<0.0001$ \\
\hline 2nd month & 107 & $11.38(6.86)$ & & $<0.0001$ \\
\hline 3rd month & 92 & $12.14(7.60)$ & & $<0.0001$ \\
\hline$>3$ months & 169 & $11.16(7.38)$ & & $<0.0001$ \\
\hline$>6$ months & 113 & $9.78(7.59)$ & & $<0.0001$ \\
\hline$>9$ months & 84 & $10.67(8.59)$ & & $<0.0001$ \\
\hline$>1$ year & 133 & $9.99(7.37)$ & & $<0.0001$ \\
\hline$>2$ years & 76 & $10.52(8.40)$ & & $<0.0001$ \\
\hline$>3$ years & 79 & $9.90(7.33)$ & & $<0.0001$ \\
\hline
\end{tabular}

Comparisons were made against baseline KMI scores using one-way ANOVA followed by Tukey's HSD post-hoc test. P-values at each follow-up are as compared to values at baseline, with a value of $\mathrm{P}<0.05$ considered to indicate a statistically significant difference. All 342 subjects received hormone treatment. KMI scores were assessed before starting MHT, and reassessed at varying intervals over the course of the treatment. Each subject's score in follow-up assessment was matched with their own baseline score. Some subjects had multiple assessments during a given interval. MHT, menopausal hormonal therapy; KMI, Kupperman Menopause Index; N/A, not available; ANOVA, analysis of variance; HSD, honestly significant difference; ${ }^{a}$ Tukey's HSD post-hoc test: P-values at each follow-up are as compared to values at baseline.

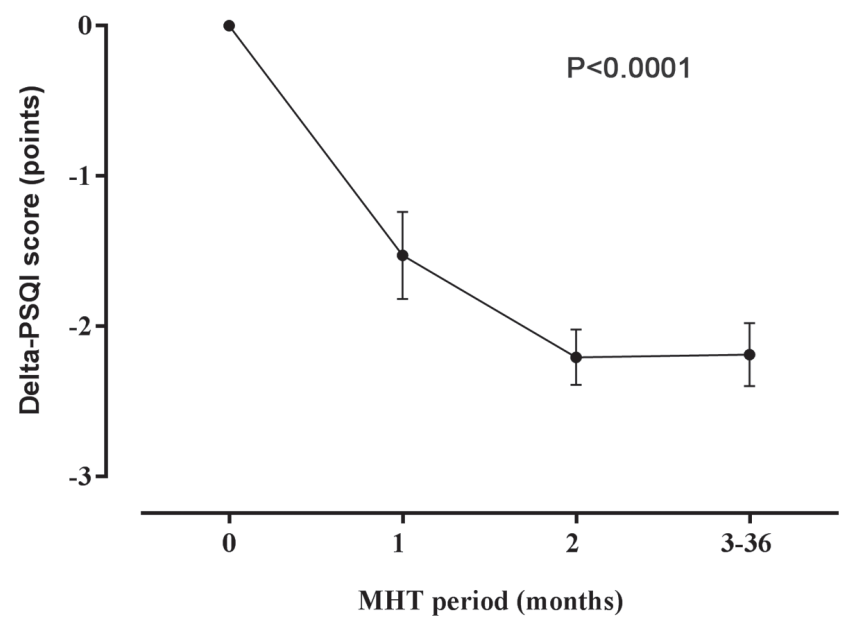

Figure 1. Effect of MHT on sleep quality. PSQI scores of study participants $(\mathrm{n}=76-342)$ were recorded prior to the commencement of MHT. The PSQI scores were then reassessed at varying intervals over the course of the treatment. Delta-PSQI indicates the difference between the initial and the follow-up score. Numbers are means $\pm \mathrm{SD} ; \mathrm{P}<0.0001$. MHT, menopausal hormonal therapy; PSQI, Pittsburgh Sleep Quality Index.

significant effect of the hormonal therapy was observed on the PSQI scores $(\mathrm{P}=0.956$; Table VI).

\section{Discussion}

In the present study, we examined the effects of hormone replacement therapy on self-reported menopausal symptoms in general, and the quality of sleep of post-menopausal women in particular. The mechanisms through which estrogen/progestin therapies may improve sleep quality are not yet well known. A previous study using rodents suggested that estrogen consolidates circadian sleep-wake rhythms in female rats (24). Alternatively, the effects of estradiol on sleep/wake cycles may

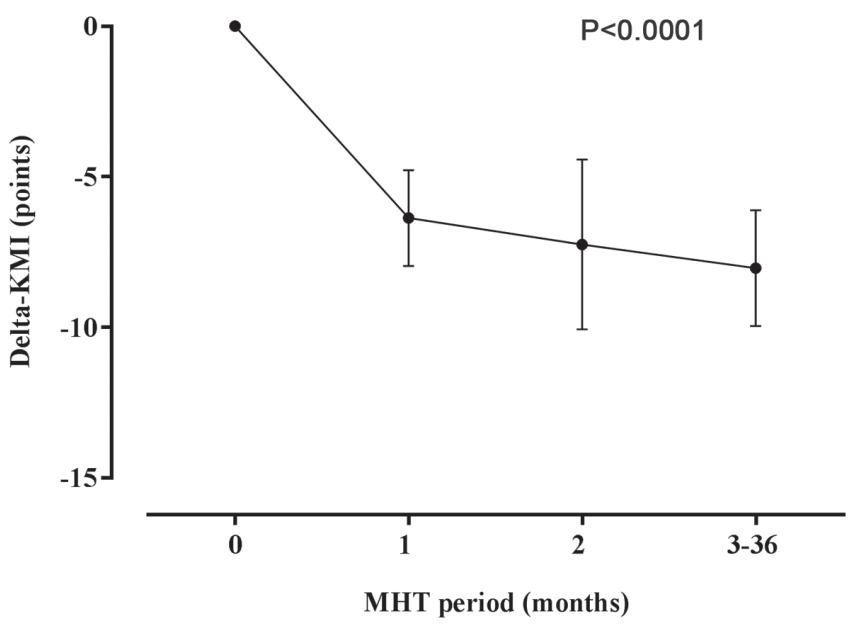

Figure 2. Effect of MHT on the KMI of the participants. KMI evaluation of menopausal symptoms was recorded prior to the commencement of MHT, and reassessed at varying intervals over the course of the treatment. Delta-KMI indicates the difference between the initial and the follow-up scores. $n=76-342$; numbers are the means \pm SD; $P<0.0001$. MHT, menopausal hormonal therapy; KMI, Kupperman Menopause Index.

be explained by a reduction in prostaglandin synthesis in the ventrolateral preoptic nucleus of the hypothalamus (25). It is also possible that estrogen therapy may improve sleep quality indirectly, by relieving nocturnal hot flashes (26). In this study, it was found that MHT induced a rapid decrease in the PSQI scores as early as 1 month after the commencement of therapy. Sleep improvement further continued through the 2nd month of MHT, resulting in a $>1.3$-fold decrease in the PSQI score compared to the baseline assessment. Notably, after the initial improvement of the PSQI scores achieved after 2 months of MHT, there was no further significant improvement in sleep quality, with the PSQI scores maintaining the same decrease of 2.19 points compared to baseline. 
Table IV. Changes in MRS scores of the in participants receiving MHT.

\begin{tabular}{|c|c|c|c|c|}
\hline MHT period & No. of participants & Mean $( \pm \mathrm{SD})$ & One-way ANOVA (P-value) & Tukey's HSD post-hoc test ${ }^{\mathrm{a}}$ (P-value) \\
\hline 0 days (baseline) & 342 & $8.66(6.17)$ & \multirow{10}{*}{$<0.0001$} & - \\
\hline 1st month & 111 & $5.10(4.67)$ & & $<0.0001$ \\
\hline 2nd month & 107 & $4.64(4.20)$ & & $<0.0001$ \\
\hline 3rd month & 92 & $4.93(4.26)$ & & $<0.0001$ \\
\hline$>3$ months & 169 & $4.93(4.32)$ & & $<0.0001$ \\
\hline$>6$ months & 113 & $4.22(3.83)$ & & $<0.0001$ \\
\hline$>9$ months & 84 & $4.32(5.68)$ & & $<0.0001$ \\
\hline$>1$ year & 133 & $3.47(3.35)$ & & $<0.0001$ \\
\hline$>2$ years & 76 & $4.04(4.09)$ & & $<0.0001$ \\
\hline$>3$ years & 79 & $4.70(4.60)$ & & $<0.0001$ \\
\hline
\end{tabular}

Comparisons were made against baseline MRS scores using one-way ANOVA followed by Tukey's HSD post-hoc test. P-values at each follow-up are as compared to values at baseline, with a value of $\mathrm{P}<0.05$ considered to indicate a statistically significant difference. All 342 subjects received hormone treatment. MRS scores were assessed before starting MHT, and reassessed at varying intervals over the course of the treatment. Each subject's score in follow-up assessment was matched with their own baseline score. Some subjects had multiple assessments during a given interval. MHT, menopausal hormonal therapy; MRS, menopause rating scale; N/A, not available; ANOVA, analysis of variance;

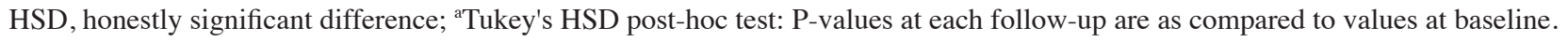

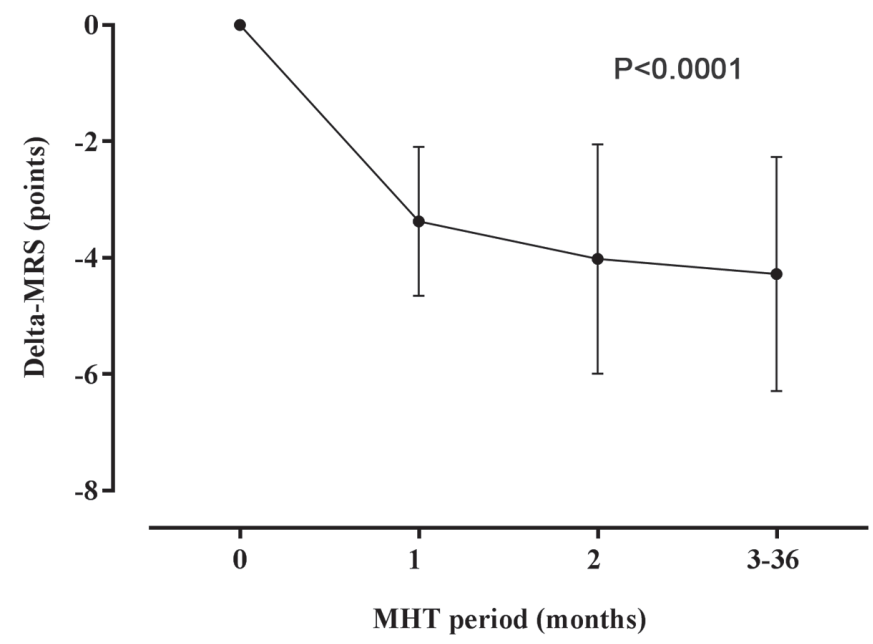

Figure 3. Effect of MHT on menopausal rating scale scores. The menopausal symptoms of the study participants ( $n=76-342)$ were assessed using MRS. Initial MRS score was prior to the commencement of MHT, and reassessed at varying intervals over the course of the treatment. Delta-MRS indicates the difference between the initial and the follow-up scores. Numbers are the means $\pm \mathrm{SD} ; \mathrm{P}<0.0001$. MHT, menopausal hormonal therapy; MRS, menopause rating scale.

In this study, the dynamics of sleep quality improvement observed in participants receiving MHT, coincided with a similar pattern of overall improvement in menopausal symptoms, as indicated by the KMI and MRS scores. It was demonstrated, that similar to the PSQI scores, patients reported the most significant decrease in the severity of menopausal symptoms during the first months of MHT. A major improvement was reported by the 2 nd month of therapy. Prolonged treatment did not result in any further alleviation of symptoms, although it rather helped to maintain the KMI and MRS scores that were decreased during the initial months of hormone replacement therapy. While the precise mechanisms

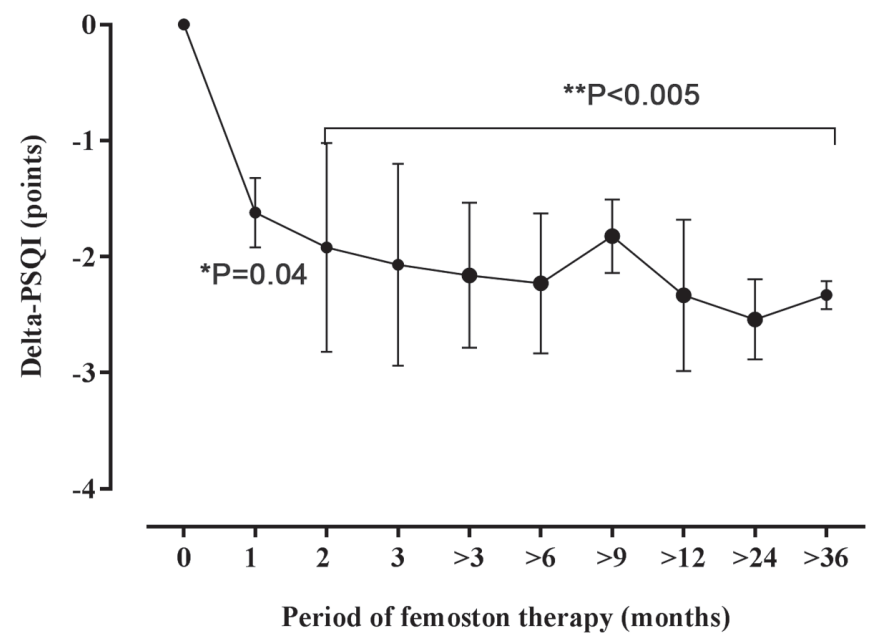

Figure 4. Effect of femoston MHT on sleep quality. Sleep quality of the subgroup of participants receiving femoston $(n=72-328)$ was assessed using the PSQI. The initial score was recorded before the beginning of femoston treatment, and reassessed at varying intervals over the course of the treatment. Delta-PSQI indicates the difference between the initial and the follow-up scores. Numbers are the means $\pm \mathrm{SD} ;{ }^{*} \mathrm{P}=0.04 ;{ }^{* *} \mathrm{P}<0.005$. MHT, menopausal hormonal therapy; PSQI, Pittsburgh Sleep Quality Index.

through which menopausal transition affect sleep quality remain unclear, previous studies have demonstrated that symptoms, such as nocturnal hot flashes, mood disorders and sleep-disordered breathing are the main contributors to a poor sleep quality $(9,27-29)$. It is possible, therefore, that the immediate partial relief of these symptoms by hormone replacement therapy may have led to a similarly rapid improvement in the sleep quality of the participants.

There is still limited evidence as regards the effects of a conventional estrogen/progestin MHT, such as femoston, on sleep quality. It has been demonstrated that progesterone may have a sedative effect (30), and estrogen is able to increase 
Table V. Changes in PSQI scores of patients receiving femoston.

\begin{tabular}{|c|c|c|c|c|}
\hline MHT period & No. of participants & Mean $( \pm$ SD $)$ & One-way ANOVA (P-value) & Tukey's HSD post-hoc test ${ }^{\mathrm{a}}$ (P-value) \\
\hline 0 days (baseline) & 328 & $9.14(4.84)$ & \multirow{10}{*}{$<0.0001$} & - \\
\hline 1 st month & 105 & $7.52(4.50)$ & & 0.04 \\
\hline 2nd month & 105 & $6.88(4.47)$ & & 0.0002 \\
\hline 3rd month & 88 & $7.07(3.98)$ & & 0.0047 \\
\hline$>3$ months & 166 & $6.90(4.30)$ & & $<0.0001$ \\
\hline$>6$ months & 110 & $6.85(4.31)$ & & 0.0001 \\
\hline$>9$ months & 76 & $7.30(4.46)$ & & 0.0417 \\
\hline$>1$ year & 129 & $6.84(4.15)$ & & $<0.0001$ \\
\hline$>2$ years & 72 & $6.61(4.52)$ & & 0.0006 \\
\hline$>3$ years & 78 & $6.81(4.72)$ & & 0.0015 \\
\hline
\end{tabular}

Comparisons were made against baseline PSQI scores using one-way ANOVA followed by Tukey's HSD post-hoc test. P-values at each follow-up are as compared to values at baseline, with a value of $\mathrm{P}<0.05$ considered to indicate a statistically significant difference. All 328 subjects received MHT with femoston. PSQI scores were assessed before starting MHT, and reassessed at varying intervals over the course of the treatment. Each subject's score in follow-up assessment was matched with their own baseline score. Some subjects had multiple assessments during a given interval. MHT, menopausal hormonal therapy; PSQI, Pittsburgh Sleep Quality Index; N/A, not available; ANOVA, analysis of

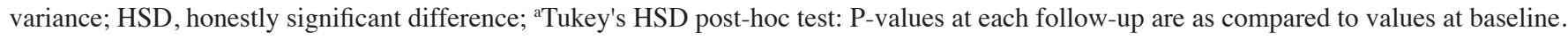

Table VI. Changes in PSQI scores of participants receiving tibolone.

\begin{tabular}{|c|c|c|c|c|}
\hline MHT period & $\mathrm{N}$ & Mean $( \pm \mathrm{SD})$ & One-way ANOVA (P-value) & Tukey's HSD post-hoc test ${ }^{\mathrm{a}}$ (P-value) \\
\hline 0 days (baseline) & 14 & $9.57(5.00)$ & \multirow{9}{*}{0.956} & - \\
\hline 1st month & 6 & $9.50(5.96)$ & & 1.09 \\
\hline 2nd month & 3 & $10.33(5.51)$ & & 1 \\
\hline 3rd month & 5 & $6.20(4.92)$ & & 0.93 \\
\hline$>3$ months & 5 & $8.80(5.02)$ & & 1 \\
\hline$>6$ months & 3 & $10.00(4.58)$ & & 1 \\
\hline$>9$ months & 8 & $8.00(4.17)$ & & 0.99 \\
\hline$>1$ year & 5 & $8.60(4.83)$ & & 1 \\
\hline$>2$ years & 4 & $8.25(3.95)$ & & 1 \\
\hline$>3$ years & 1 & 14 (N/A) & - & - \\
\hline
\end{tabular}

Comparisons were made against baseline PSQI scores using one-way ANOVA followed by Tukey's HSD post-hoc test. P-values at each follow-up are as compared to values at baseline, with a value of $\mathrm{P}<0.05$ considered to indicate a statistically significant difference. All 14 subjects were treated with tibolone. PSQI scores were assessed before starting MHT, and reassessed at varying intervals over the course of the treatment. Each subject's score in follow-up assessment was matched with their own baseline score. Due to the low number of cases where tibolone was administered, statistical analysis could not be applied to some intervals and have low validity for others. MHT, menopausal hormonal therapy; PSQI, Pittsburgh Sleep Quality Index; N/A, not available; ANOVA, analysis of variance; HSD, honestly significant difference; ${ }^{a}$ Tukey's HSD post-hoc test: P-values at each follow-up are as compared to values at baseline.

overall sleep time, and decrease sleep onset latency (31), and awakening after sleep onset (32). In this study, femoston was found to have a marked and rapid effect on self-reported sleep quality, with the PSQI score decreasing 1.32-fold during the first 2 months of the treatment. This decrease was maintained throughout the 3 years of the study period, suggesting that estrogen/progestin MHT results in a stable improvement of sleep quality. Of note, MHT with tibolone did not improve sleep quality in a small group of participants with a previous history of breast disease. Tibolone is a synthetic steroid that has progestogenic, androgenic, and estrogenic effects, shown to improve insomnia via stimulation of the production and release of $\beta$-endorphin $(33,34)$. It is possible that the lack of an effect of tibolone on sleep quality in this study is related to an insufficient number of participants receiving this MHT. Better designed, larger clinical studies are thus required to examine the efficiency of tibolone in improving the sleep quality of menopausal women.

In conclusion, this study found that hormone replacement therapy had an immediate and long-lasting positive effect on sleep quality and on the general well-being of women 
experiencing menopausal symptoms. While a conventional estrogen/progestin MHT with femoston resulted in a marked improvement of sleep quality, tibolone treatment had no effect on the PSQI score. Further clinical studies with larger patient groups are required to compare the effects of both MHT regiments on sleep quality.

\section{Acknowledgements}

Not applicable.

\section{Funding}

No funding was received.

\section{Availability of data and materials}

The datasets used and/or analyzed during the current study are available from the corresponding author on reasonable request.

\section{Authors' contributions}

CL and XY conceived and designed the study. CL, LW and XS provided the study materials or patient data and were responsible for the collection and assembly of the data, data analysis and interpretation. CL was involved in the writing of the manuscript. XY was involved in the editing of the manuscript. All authors have read and approved the final manuscript.

\section{Ethics approval and consent to participate}

The Ethics Committee of the Maternity and Child Health Care of Zaozhuang approved this retrospective medical record review (approval no. 2016006).

\section{Patient consent for publication}

Not applicable.

\section{Competing interests}

The authors declare that they have no competing interests.

\section{References}

1. Jehan S, Masters-Isarilov A, Salifu I, Zizi F, Jean-Louis G Pandi-Perumal SR, Gupta R, Brzezinski A and McFarlane SI: Sleep Disorders in Postmenopausal Women. J Sleep Disord Ther 4: 4, 2015.

2. Al-Safi ZA and Santoro N: Menopausal hormone therapy and menopausal symptoms. Fertil Steril 101: 905-915, 2014.

3. Potthoff P, Heinemann LA, Schneider HP, Rosemeier HP and Hauser GA: The Menopause Rating Scale (MRS II) Methodological standardization in the German population. Zentralbl Gynakol 122: 280-286, 2000 (In German).

4. Schneider HP, Heinemann LA, Rosemeier HP, Potthoff P and Behre HM: The Menopause Rating Scale (MRS): Reliability of scores of menopausal complaints. Climacteric 3: 59-64, 2000.

5. Kupperman HS, Blatt MH, Wiesbader $\mathrm{H}$ and Filler W: Comparative clinical evaluation of estrogenic preparations by the menopausal and amenorrheal indices. J Clin Endocrinol Metab 13: 688-703, 1953.

6. Tao M, Shao H, Li C and Teng Y: Correlation between the modified Kupperman Index and the Menopause Rating Scale in Chinese women. Patient Prefer Adherence 7: 223-229, 2013.
7. Mitchell ES and Woods NF: Symptom experiences of midlife women: Observations from the Seattle Midlife Women's Health Study. Maturitas 25: 1-10, 1996.

8. Lai JN, Chen HJ, Chen CM, Chen PC and Wang JD: Quality of life and climacteric complaints amongst women seeking medical advice in Taiwan: Assessment using the WHOQOL-BREF questionnaire. Climacteric 9: 119-128, 2006.

9. Dennerstein L, Dudley EC, Hopper JL, Guthrie JR and Burger HG: A prospective population-based study of menopausal symptoms. Obstet Gynecol 96: 351-358, 2000.

10. Kawada T, Yosiaki S, Yasuo K and Suzuki S: Population study on the prevalence of insomnia and insomnia-related factors among Japanese women. Sleep Med 4: 563-567, 2003.

11. Pan HA, Wu MH, Hsu CC, Yao BL and Huang KE: The perception of menopause among women in Taiwan. Maturitas 41: 269-274, 2002.

12. Shade MY, Berger AM, Dizona PJ, Pozehl BJ and Pullen CH: Sleep and health-related factors in overweight and obese rural women in a randomized controlled trial. J Behav Med 39: 386-397, 2016.

13. Chen JH, Waite LJ and Lauderdale DS: Marriage, Relationship Quality, and Sleep among U.S. Older Adults. J Health Soc Behav 56: 356-377, 2015.

14. Lampio L, Saaresranta T, Engblom J, Polo O and Polo-Kantola P Predictors of sleep disturbance in menopausal transition. Maturitas 94: 137-142, 2016.

15. Luyster FS, Strollo PJ Jr, Zee PC and Walsh JK; Boards of Directors of the American Academy of Sleep Medicine and the Sleep Research Society: Sleep: A health imperative. Sleep (Basel) 35: 727-734, 2012.

16. Lobo RA: Where are we 10 years after the Women's Health Initiative? J Clin Endocrinol Metab 98: 1771-1780, 2013.

17. Formoso G, Perrone E, Maltoni S, Balduzzi S, D'Amico R, Bassi C, Basevi V, Marata AM, Magrini N and Maestri E: Short and long term effects of tibolone in postmenopausal women. Cochrane Database Syst Rev 2012: CD008536, 2012.

18. Bruyneel M: Sleep disturbances in menopausal women: Aetiology and practical aspects. Maturitas 81: 406-409, 2015

19. Cintron D, Lipford M, Larrea-Mantilla L, Spencer-Bonilla G, Lloyd R, Gionfriddo MR, Gunjal S, Farrell AM, Miller VM and Murad MH: Efficacy of menopausal hormone therapy on sleep quality: Systematic review and meta-analysis. Endocrine 55: 702-711, 2017.

20. Backhaus J, Junghanns K, Broocks A, Riemann D and Hohagen F: Test-retest reliability and validity of the Pittsburgh Sleep Quality Index in primary insomnia. J Psychosom Res 53: 737-740, 2002.

21. Mollayeva T, Thurairajah P, Burton K, Mollayeva S, Shapiro CM and Colantonio A: The Pittsburgh sleep quality index as a screening tool for sleep dysfunction in clinical and non-clinical samples: A systematic review and meta-analysis. Sleep Med Rev 25: 52-73, 2016.

22. Devine EB, Hakim Z and Green J: A systematic review of patient-reported outcome instruments measuring sleep dysfunction in adults. Pharmacoeconomics 23: 889-912, 2005.

23. Buysse DJ, Reynolds CF III, Monk TH, Berman SR and Kupfer DJ: The Pittsburgh Sleep Quality Index: A new instrument for psychiatric practice and research. Psychiatry Res 28: 193-213, 1989.

24. Schwartz MD and Mong JA: Estradiol modulates recovery of REM sleep in a time-of-day-dependent manner. Am J Physiol Regul Integr Comp Physiol 305: R271-R280, 2013.

25. Mong JA, Devidze N, Goodwillie A and Pfaff DW: Reduction of lipocalin-type prostaglandin D synthase in the preoptic area of female mice mimics estradiol effects on arousal and sex behavior. Proc Natl Acad Sci USA 100: 15206-15211, 2003.

26. Polo-Kantola P, Erkkola R, Helenius H, Irjala K and Polo O: When does estrogen replacement therapy improve sleep quality? Am J Obstet Gynecol 178: 1002-1009, 1998.

27. Ohayon MM: Severe hot flashes are associated with chronic insomnia. Arch Intern Med 166: 1262-1268, 2006.

28. Shahar E, Redline S, Young T, Boland LL, Baldwin CM, Nieto FJ, O'Connor GT, Rapoport DM and Robbins JA: Hormone replacement therapy and sleep-disordered breathing. Am J Respir Crit Care Med 167: 1186-1192, 2003.

29. Krystal AD, Edinger J, Wohlgemuth W and Marsh GR: Sleep in peri-menopausal and post-menopausal women. Sleep Med Rev 2: 243-253, 1998.

30. Manber R and Armitage R: Sex, steroids, and sleep: A review. Sleep 22: 540-555, 1999. 
31. Schiff I, Regestein Q, Tulchinsky D and Ryan KJ: Effects of estrogens on sleep and psychological state of hypogonadal women. JAMA 242: 2405-4, 1979.

32. Landis CA and Moe KE: Sleep and menopause. Nurs Clin North Am 39: 97-115, 2004.

33. Genazzani AR, Facchinetti F, Ricci-Danero MG, Parrini D, Petraglia F, La Rosa R and D'Antona N: Beta-lipotropin and beta-endorphin in physiological and surgical menopause. J Endocrinol Invest 4: 375-378, 1981.
34. Taskin O, Gökdeniz R, Yalcinoglu A, Buhur A, Burak F, Atmaca R and Ozekici U: Placebo-controlled cross-over study of effects of tibolone on premenstrual symptoms and peripheral beta-endorphin concentrations in premenstrual syndrome. Hum Reprod 13: 2402-2405, 1998.

(i) (3) () This work is licensed under a Creative Commons c) International (CC BY-NC-ND 4.0) License. 